\title{
Ribosomal proteins and ribosomal pseudogenes are differentially expressed by medullary and cortical epithelial cells of the thymus.
}

\section{Shahan Mamoor}

San Diego, CA 92101

shahanmamoor@gmail.com

The thymus is a unique organ with the ability to impart the concept of self-tolerance, or immunological privilege to developing lymphocytes $(1,2)$. It possesses anatomical compartmentalization in the form of a medulla and cortex (3). To understand the transcriptional behavior of the medullary (mTEC) and cortical epithelial cells (cTEC) of the thymus as they most differ from each other, we performed global differential gene expression profiling using a public microarray dataset of each cell type, isolated from the mouse thymus (4). These analyses revealed that eleven unique ribosomal proteins and pseudogenes were among the most differentially expressed genes when comparing the mTEC transcriptome with the cTEC transcriptome. These data suggest a potential cell-type specific role for these ribosomal subunits and pseudogenes in the epithelial cells of the thymus.

Keywords: thymus, medullary epithelial cells, cortical epithelial cells, cTEC, mTEC(hi), mTEC(lo), ribosome, ribosomal subunits, ribosomal pseudogene 


\section{Introduction}

The thymus is a unique organ with the ability to educate developing lymphocytes what it means to be self by presenting them with antigen that is generally otherwise restricted to the periphery (peripheral tissue antigen) (1-3). Antigen presentation for lymphocyte tolerance instruction occurs in an anatomically regulated fashion, with thymic epithelial cells in the cortex and medulla, termed CTEC and MTEC (3), and is controlled in large part by expression of the Class II major histocompatibility complex peptide antigen receptor MHC-II $(5,6)$. mTEC expressing high levels of MHC-II (mTEChi) also express relatively higher levels of the autoimmune regulator Aire, which induces promiscuous expression of tissue-restricted antigen for self-antigen presentation $(5,6)$.

Here we used a public microarray dataset to perform global differential gene expression analysis with a cross-comparison of the mTEChi, mTEClo, and cTEC transcriptomes from adult murine thymus. This transcriptome study revealed that ribosomal protein subunits and ribosomal pseudogenes are among the most differentially expressed genes when comparing these cell types and suggests a potential role for cell-type specific ribosomal subunit function in immunologically active cells of the thymus.

\section{Methods}

GSE89965 was utilized for this differential gene expression analysis, performed using GEO2R. This public dataset was generated by the authors using microarray (Affymetrix Mouse Gene 1-ST array) analysis of Aire-GFP, EpCAM+Ly51+ cells (cTEC), Aire-GFP Epcam+MHCII(hi) cells (mTEChi), and Aire-GFP Epcam+MHC-II(lo) cells (mTEClo); $n=4$ from mTEChi and mTEClo; $n=3$ from cTEC, from C57BI/6 Adig mice 6 weeks of age (adult). No adjustment was made to $p$-values, and there was no log-transformation applied to the data. The NCBI generated category of platform annotation was used. An unpaired two-tailed t-test with Welch's correction was utilized to determine the statistical significance of the RNA expression values between each cell type. For statistical test to determine the significance of Rps2 
pseudogene expression values across the three cell types, a one-way ANOVA with multiple comparisons was performed. Data are from three analyses; comparing mTEChi to mTEClo, comparing mTEChi to cTEC, and comparing mTEClo to cTEC.

\section{Results}

Multiple ribosomal subunits and ribosomal pseudogenes are differentially expressed when comparing the transcriptomes of mTEChi to cTEC.

We performed comparative global gene expression profiling to determine the most differentially expressed genes between the transcriptomes of mTEChi and cTEC using a public dataset of microarray expression data from flow cytometry sorted EpCAM+MHC-II+ and EpCAM+Ly51+ cells. This systems-level analysis revealed that among the messages whose expression was most different between the mTEChi and cTEC transcriptomes were six ribosomal subunits and five ribosomal pseudogenes.

Ribosomal protein S18, Rps18 was ranked 40 out of 34760 transcripts detected by microarray in this dataset (Table 1; $\mathrm{p}=0.000003$ ); ribosomal protein L23, Rpl23 was ranked 179 $(p=0.0000192)$, ribosomal protein large P0, Rplp0 was ranked 190 (Table 1; $p=0.0000203$ ); ribosomal protein S7 was ranked 192 (Table 1; $p=0.0000206$ ); ribosomal protein L6 was ranked 215 ( $p=0.0000254) ;$ ribosomal protein S10 was ranked 227 (Table 1; $p=0.0000268$ ); and ribosomal protein L3, Rpl3 was ranked 250 (Table 1; $p=0.0000305$ ).

The ribosomal protein L7a pseudogene, or Rpl7a (Gm5766) was ranked 66 out of 34760 transcripts detected (Table $1 ; p=0.00000544$ ); the ribosomal protein large P0 pseudogene Rplp0 (Gm5779) was ranked 103 (Table 1; p=0.0000941); the ribosomal protein S2 pseudogene, Rps2(Gm10653) was ranked 168 (Table 1; p=0.0000173), and ribosomal protein S23 pseudogene, Rps23 was ranked 267 (Table 1; p=0.0000326).

The expression of all of these genes was significantly higher in mTEChi, except for the Rps2 pseudogene, which was expressed at significantly higher levels in cTEC (Figure 1B, $\mathrm{p}=0.0025)$. For all genes expressed at significantly higher levels in mTEChi, $\mathrm{p}$ was calculated to 
be less than or equal to 0.0001 except for Rpl6 $p=0.0002)$, the Rpl7a pseudogene $(p=0.0039)$, and the Rpl3 pseudogene $(p=0.0004)$.

Overall, five ribosomal subunits and four ribosomal subunit pseudogenes were differentially expressed at the global level in mTEChi when comparing its transcriptome to that of cTEC, and their expressional levels were significantly higher in mTEChi than in cTEC.

Specific ribosomal subunits are differentially expressed, and at significantly higher levels in the mTEClo transcriptome when compared to cTEC.

We continued our systems-level study of mTEC and cTEC transcriptional behavior by comparing microarray data from mTEClo and cTEC ( $n=4$ for mTEC $^{l o}$ and $n=3$ for cTEC).

Here, four ribosomal subunits were significantly differentially expressed between mTEClo and cTEC at the global level, and three were expressed at significantly higher levels in mTEClo. Rps9 (rank 20 out of 34760), Rps13 (rank 95), and Rpl23a (rank 226) were expressed at significantly higher levels in mTEClo,(Figure 2; Rps9: $p=0.0007 ;$ Rps13: $p=0.0015$; and Rpl23a: $p=0.0028$ ) while Rps10a (rank 208) was expressed at significantly higher levels in cTEC (Figure 2; Rps10a: $p=0.0031$ ).

The only pseudogene among the two hundred and fifty most differentially expressed genes in this analysis, ribosomal protein S2 pseudogene Rps2, was expressed at significantly higher levels in cTEC (Figure 2; $p=0.0091$ ).

The Rps2 pseudogene is differentially expressed when comparing the transcriptomes of mTEC hi to $\mathrm{mTEC}^{\mathrm{lo}}$, and at significantly higher levels in mTEClo

When comparing the transcriptomes of $\mathrm{MTEC}^{\text {hi }}$ and $\mathrm{mTEC}^{\mathrm{lo}}$, the only ribosomal subunit or subunit pseudogene that was differentially expressed and within the top 250 most differentially expressed genes globally was the Rps2 pseudogene (Table $1 ; p=0.000133$ ). Rps2 pseudogene was the 176th most differentially expressed gene between the transcriptomes of

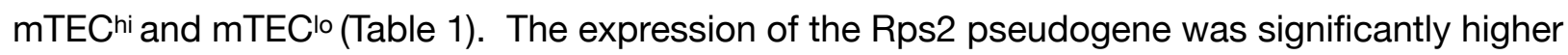

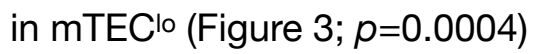


Rps2 pseudogene is differentially expressed in the epithelial cells of the thymus and graded across cell type.

The only gene that was differentially expressed at the threshold of $250 / 34760$ total transcripts detected by the microarray dataset utilized in this study was the Rps2 pseudogene (Figure 4). The expression of the Rps2 pseudogene was significantly higher in cTEC than in mTEClo and mTEChi, and was significantly higher in mTEClo than in mTEChi.

\section{Discussion}

To better understand the transcriptional behavior of the epithelial cells of the thymus, in the cortex and the medulla, we compared the transcriptomes of cTEC, mTEClo and mTEChi (expressing low or high levels of the MHC Class II receptor), isolated from the mouse thymus

(4) and asked which RNA messages were most significantly different, globally, in terms of their expression levels. We found that multiple ribosome subunits, both large (Rpl) and small (Rps) (7) were among the most differentially expressed genes in these thymic cell types.

The Rps2 pseudogene was the one ribosomal-type gene that was differentially expressed across all three comparisons at the threshold of our analysis (two hundred and fifty out of 34760). The expression of the Rps2 pseudogene may serve some critical function in these cell types that is appropriately graded, or it may be a reflection of some other cell-type specific process that displays a cell-type specific frequency or quality. Cowan et al. recently reported that Myc controls thymus size in the fetal thymus by enhancing ribosomal biogenesis in fetal TEC (8). This study did not isolate or study cells based on expression of MHC-Class II, nor did it report any role for or pattern of expression of ribosomal pseudogenes in the TEC cells of the thymus as we do here.

In conclusion, transcripts encoding ribosomal protein subunits and and non-coding ribosomal pseudogene transcripts are significant components of the transcriptome of thymic epithelial cells, in both the medulla and cortex. Most of these ribosomal subunits were expressed at significantly higher levels in the medulla, except for Rpl10a. Global differential expression of ribosomal protein $S$ and ribosomal protein $L$ subunits could potentially reflect 
cell-type specific functions for these ribosomal subunits in antigen presentation during Airedependent education during of self during lymphocyte development. A function for ribosomal subunit pseudogenes (9) in the thymus is not clear but may similarly reflect some mechanisms required for regulation of gene expression during Aire-dependent training of tolerance by the medullary epithelial cells of the thymus by the Class II MHC antigen receptor, and should be the focus of future study. 


\section{References}

1. Anderson, M.S., Venanzi, E.S., Klein, L., Chen, Z., Berzins, S.P., Turley, S.J., Von Boehmer, H., Bronson, R., Dierich, A., Benoist, C. and Mathis, D., 2002. Projection of an immunological self shadow within the thymus by the aire protein. Science, 298(5597), pp. 1395-1401.

2. Hogquist, K.A., Baldwin, T.A. and Jameson, S.C., 2005. Central tolerance: learning selfcontrol in the thymus. Nature Reviews Immunology, 5(10), p.772.

3. Rossi, S.W., Jenkinson, W.E., Anderson, G. and Jenkinson, E.J., 2006. Clonal analysis reveals a common progenitor for thymic cortical and medullary epithelium. Nature, 441(7096), p.988.

4. Jakub Abramson, Immunology, Weizmann Institute; PRJNA354356; GEO: GSE89965.

5. Gray, D., Abramson, J., Benoist, C. and Mathis, D., 2007. Proliferative arrest and rapid turnover of thymic epithelial cells expressing Aire. Journal of Experimental

Medicine, 204(11), pp.2521-2528.

6. Hinterberger, M., Aichinger, M., Da Costa, O.P., Voehringer, D., Hoffmann, R. and Klein, L., 2010. Autonomous role of medullary thymic epithelial cells in central CD4+ T cell tolerance. Nature immunology, 11(6), p.512.

7. Lake, J.A., 1976. Ribosome structure determined by electron microscopy of Escherichia coli small subunits, large subunits and monomeric ribosomes. Journal of molecular biology, 105(1), pp.131-159.

8. Cowan, J.E., Malin, J., Zhao, Y., Seedhom, M.O., Harly, C., Ohigashi, I., Kelly, M., Takahama, Y., Yewdell, J.W., Cam, M. and Bhandoola, A., 2019. Myc controls a distinct transcriptional program in fetal thymic epithelial cells that determines thymus growth. Nature communications, 10(1), pp.1-14.

9. Zhang, Z., Harrison, P. and Gerstein, M., 2002. Identification and analysis of over 2000 ribosomal protein pseudogenes in the human genome. Genome research, 12(10), pp. 1466-1482. 


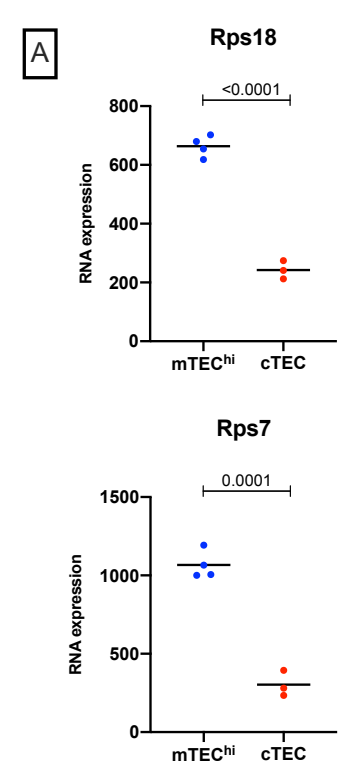

B

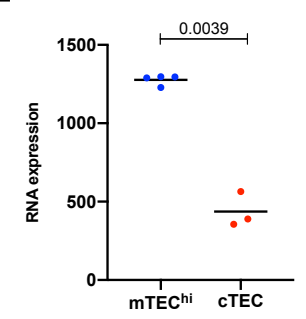

Gm5789//Rpl3 pseudogene

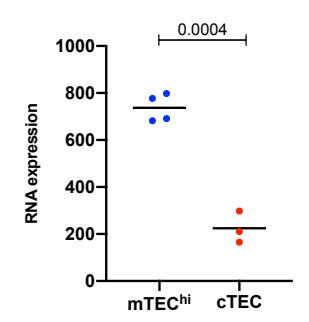

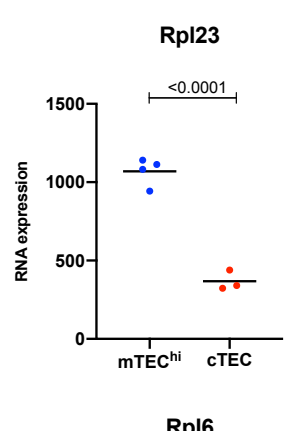

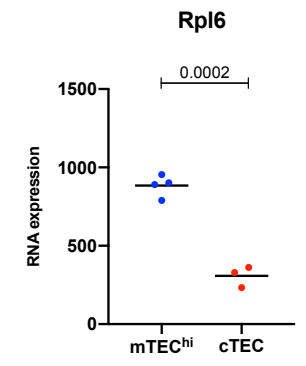

Gm5779//Rplp0 pseudogene

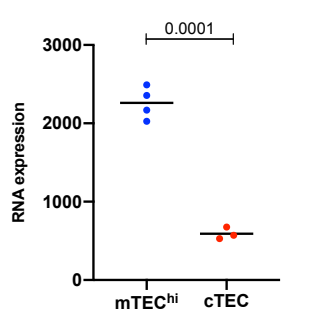

Gm8624//Rps23 pseudogene

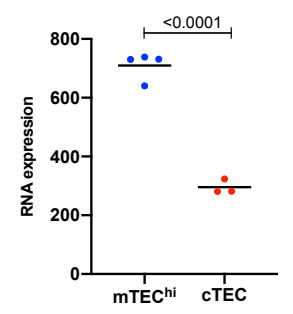

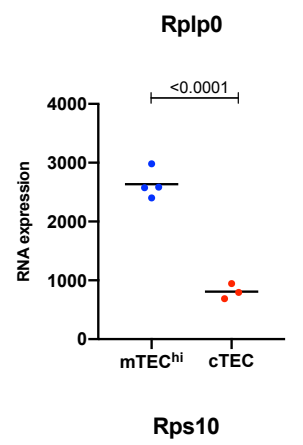

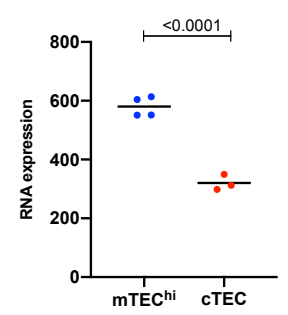

Gm10653//Rps2 pseudogene

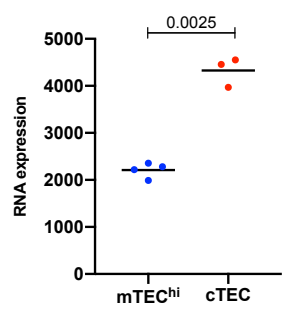

Figure 1: Multiple ribosomal subunits and ribosomal pseudogenes are differentially expressed, and at significantly higher levels in the $\mathrm{MTEC}^{\text {hi }}$ transcriptome when compared to cTEC.

Figure 1A: The RNA expression value of six ribosomal subunits is shown with each dot representing one pool of thymic cells isolated from mice $(n=4$ for $m$ TEChi (blue) and $n=3$ for cTEC (red)). The black bar represents the mean value. The statistical significance of the difference in expression values between the two categories was calculated using a two-tailed unpaired t-test with Welch's correction, and displayed on each graph as a p-value.

Figure 1B: The RNA expression value of five ribosomal subunit pseudogenes is shown with each dot representing one pool of thymic cells isolated from mice ( $n=4$ for mTEChi (blue) and $\mathrm{n}=3$ for cTEC (red)). The black bar represents the mean value. The statistical significance of the difference in expression values between the two categories was calculated using a twotailed unpaired t-test with Welch's correction, and displayed on each graph as a p-value. 
A
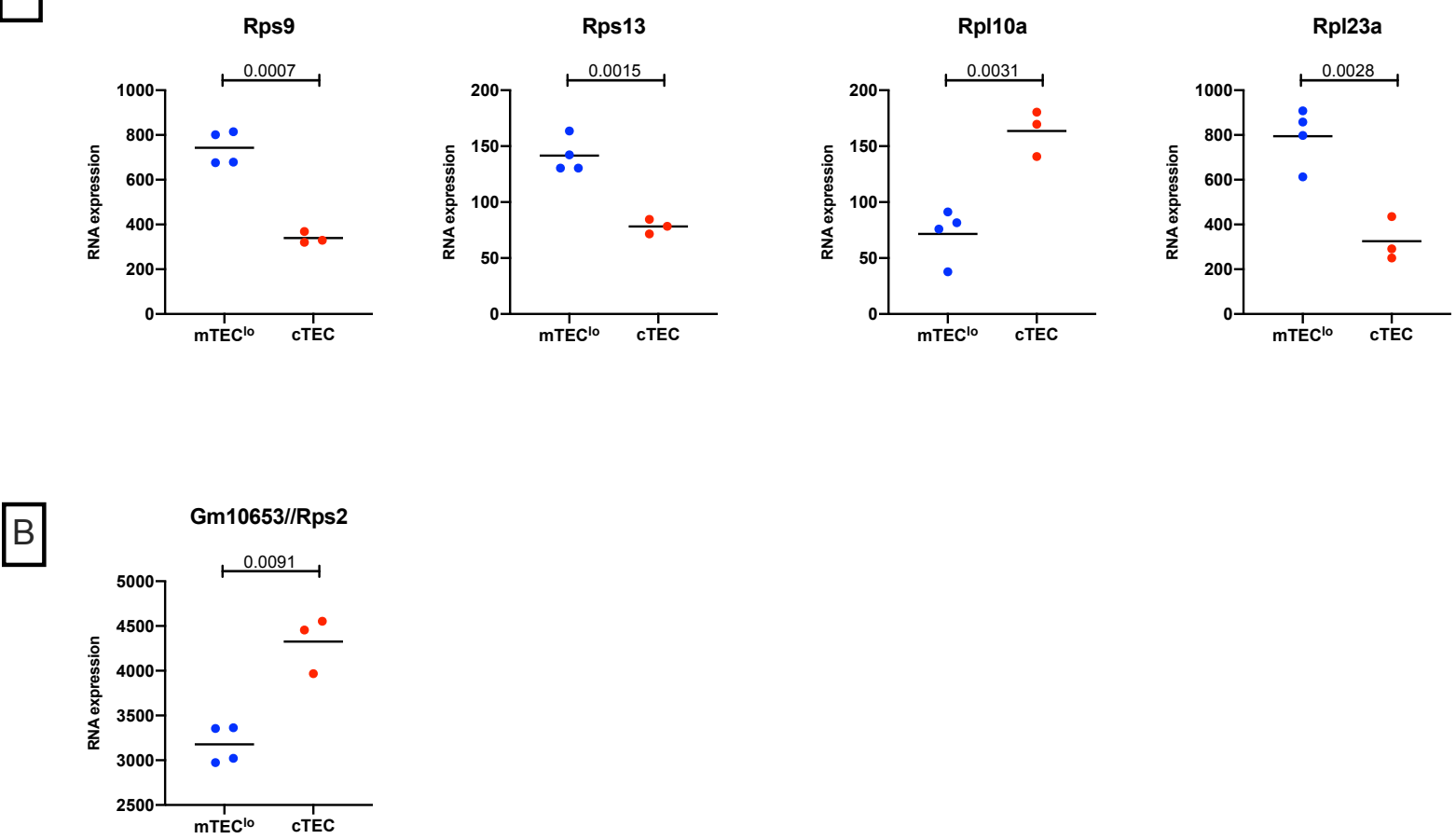

Figure 2: Multiple ribosomal subunits are differentially expressed, and at significantly higher levels in the mTEClo transcriptome when compared to cTEC.

Figure 2A: The RNA expression value of four ribosomal subunits is shown with each dot

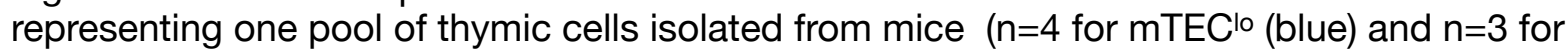
cTEC (red)). The black bar represents the mean value. The statistical significance of the difference in expression values between the two categories was calculated using a two-tailed unpaired t-test with Welch's correction, and displayed on each graph as a p-value.

Figure 2B: The RNA expression value of five ribosomal subunit pseudogenes is shown with each dot representing one pool of thymic cells isolated from mice ( $n=4$ for mTEClo (blue) and $\mathrm{n}=3$ for cTEC (red)). The black bar represents the mean value. The statistical significance of the difference in expression values between the two categories was calculated using a twotailed unpaired t-test with Welch's correction, and displayed on each graph as a p-value. 


\section{Gm10653///Rps2}

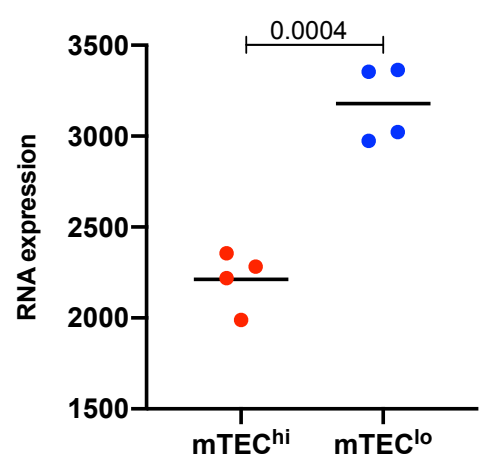

Figure 3: The Rps2 pseudogene is differentially expressed when comparing the

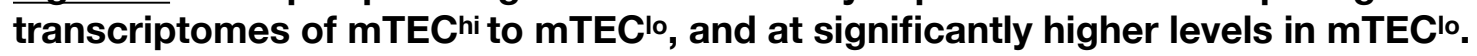

The mean expression level of the Rps2 pseudogene is marked with a black bar ( $\mathrm{n}=4$ for mTEChi (red), and $n=4$ for $\mathrm{mTEC}^{\mathrm{lo}}$ (blue)). The statistical significance of the difference in expression values between the two categories was calculated using a two-tailed unpaired t-test with Welch's correction, and displayed on the graph as a $p$-value. 


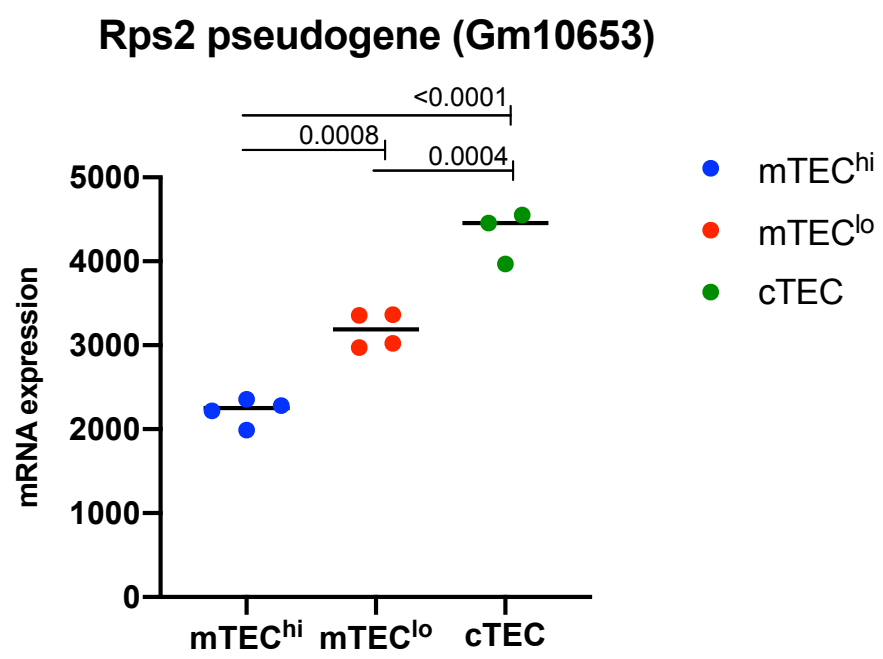

Figure 4: The Rps2 pseudogene is differentially expressed when comparing the transcriptomes of $\mathrm{mTEChi}$ to $\mathrm{mTEClo}$, and at significantly higher levels in MTEClo

The mean expression level of the Rps2 pseudogene is marked with a black bar ( $\mathrm{n}=4$ for mTEChi (blue), and $n=4$ for $m$ TEClo (red) and and $n=3$ for cTEC). The statistical significance of the difference in expression values between the two categories was calculated using a one-way ANOVA with multiple comparisons, and displayed on the graph as a $p$-value. 
Table 1

\begin{tabular}{|c|c|c|c|c|c|c|}
\hline Rank & Gene Symbol & Gene Name & p-value & $\mathbf{t}$ & B & ID \\
\hline 40 & Rps18 & ribosomal protein S18 & 0.000003 & -17.4263101 & -3.34 & 10581535 \\
\hline 66 & Gm5766///Rpl7a & $\begin{array}{l}\text { ribosomal protein L7a pseudogene///ribosomal } \\
\text { protein L7A }\end{array}$ & 0.00000544 & -15.7084304 & -3.34 & 10603172 \\
\hline 103 & Gm5779///Rplp0 & $\begin{array}{l}\text { ribosomal protein, large, P0 pseudogene/// } \\
\text { ribosomal protein, large, PO }\end{array}$ & 0.0000941 & -14.2664069 & -3.35 & 10581027 \\
\hline 168 & Gm10653///Rps2 & $\begin{array}{l}\text { ribsomal protein S2 pseudogene///ribosomal } \\
\text { protein S2 }\end{array}$ & 0.0000173 & 12.8112523 & -3.37 & 10402314 \\
\hline 179 & Rpl23 & ribosomal protein L23 & 0.0000192 & -12.5773205 & -3.37 & 10545125 \\
\hline 190 & Rplp0 & ribosomal protein, large, P0 & 0.0000203 & -12.4583289 & -3.37 & 10524718 \\
\hline 192 & Rps7 & ribosomal protein S7 & 0.0000206 & -12.4228698 & -3.37 & 10607391 \\
\hline 215 & Rpl6 & ribosomal protein L6 & 0.0000254 & -11.970194 & -3.37 & 10525187 \\
\hline 227 & Rps10 & ribosomal protein S10 & 0.0000268 & -11.8564181 & -3.38 & 10449370 \\
\hline 250 & Gm5879///Rpl3 & predicted gene $5879 / / /$ ribosomal protein L3 & 0.0000305 & -11.5869932 & -3.38 & 10600324 \\
\hline 267 & Gm8624///Rps23 & $\begin{array}{l}\text { ribosomal protein } \mathrm{S} 23 \text { pseudogene/// } \\
\text { ribosomal protein S23 }\end{array}$ & 0.0000326 & 11.4523442 & -3.38 & 10406541 \\
\hline Rank & Gene Symbol & Gene Name & p-value & $\mathbf{t}$ & B & ID \\
\hline 20 & Rps9 & ribosomal protein S9 & 0.00010265 & -9.343295 & -3.72 & 10549588 \\
\hline 95 & Rps13 & ribosomal protein $\mathrm{S} 13$ & 0.00057798 & -6.775544 & -3.79 & 10567163 \\
\hline 126 & Gm10653///Rps2 & $\begin{array}{l}\text { ribsomal protein S2 pseudogene///ribosomal } \\
\text { protein S2 }\end{array}$ & 0.00083082 & 6.319075 & -3.81 & 10402314 \\
\hline 208 & Rpl10a & ribosomal protein L10A & 0.00134125 & 5.75362 & -3.83 & 10407535 \\
\hline 226 & Rpl23a & ribosomal protein L23A & 0.00148276 & -5.640193 & -3.84 & 10598103 \\
\hline Rank & Gene Symbol & Gene Name & p-value & $\mathbf{t}$ & B & ID \\
\hline 176 & Gm10653///Rps2 & $\begin{array}{l}\text { ribsomal protein S2 pseudogene///ribosomal } \\
\text { protein S2 }\end{array}$ & 0.000133 & 7.795409 & -3.05 & 10402314 \\
\hline
\end{tabular}

Table 1: Multiple ribosomal subunits and ribosomal pseudogenes are differentially expressed in the MTEC and CTEC transcriptomes.

Shown is the rank of differential expression relative to the 34760 total transcripts detected in each analysis, gene symbol, gene name, $p$-value with respect to global differential expression between each comparison, $t$, a moderated t-statistic, $B$, the log-odds probability of differential expression in the given comparison, and NCBI ID (all provided by GEO2R).

Table 1A: mTEChi vs cTEC

Table 1B: mTEClo vs cTEC

Table 1C: mTEChi vs mTEClo 